\title{
Coupling of D2R Short but not D2R Long receptor isoform to the Rho/ROCK signaling pathway renders striatal neurons vulnerable to mutant huntingtin
}

\author{
Beatriz Galan-Rodriguez, ${ }^{1,2}$ Elodie Martin, ${ }^{3}$ Emmanuel Brouillet, ${ }^{4,5}$ Nicole Déglon, ${ }^{6,7}$ Sandrine Betuing ${ }^{1,8,9, *}$ and \\ Jocelyne Caboche ${ }^{1,8,9, *}$ \\ ${ }^{1}$ UMRS-INSERM1130, Neurosciences Paris Seine, Paris, France \\ ${ }^{2}$ Departamento de Fisiología Médica y Biofísica, Universidad de Sevilla, Sevilla, Spain \\ ${ }^{3}$ INSERM UMRS_1127/UPMC/CNRS UMR7225, Institut du Cerveau et de la Moelle, Hôpital Pitié-Salpêtrière, Paris, France \\ ${ }^{4} \mathrm{CEA}$, DSV, I'BM, Molecular Imaging Research Center (MIRCen), Fontenay-aux-Roses, France \\ ${ }^{5}$ Neurodegenerative Diseases Laboratory, CNRS CEA URA 2210, Fontenay-aux-Roses, France \\ ${ }^{6}$ Department of Clinical Neurosciences (DNC), Laboratory of Cellular and Molecular Neurotherapies (LNCM), Lausanne \\ University Medical School (CHUV), Lausanne, Switzerland \\ ${ }^{7}$ Neuroscience Research Center (CRN), Laboratory of Cellular and Molecular Neurotherapies (LNCM), Lausanne University \\ Medical School (CHUV), Lausanne, Switzerland \\ ${ }^{8}$ UMR CNRS-8246, Paris, France \\ ${ }^{9}$ Sorbonne Université, UM119, Université Pierre and Marie Curie-Paris 6, 9 quai Saint Bernard, 75005 Paris, France
}

Keywords: aggregates, D2 receptor isoforms, Huntington's disease, intracellular signaling, striatal death

Edited by Paul Bolam

Received 27 April 2016, revised 9 September 2016, accepted 21 September 2016

\begin{abstract}
Huntington's disease, an inherited neurodegenerative disorder, results from abnormal polyglutamine extension in the N-terminal region of the huntingtin protein. This mutation causes preferential degeneration of striatal projection neurons. We previously demonstrated, in vitro, that dopaminergic D2 receptor stimulation acted in synergy with expanded huntingtin to increase aggregates formation and striatal death through activation of the Rho/ROCK signaling pathway. In vivo, in a lentiviral-mediated model of expanded huntingtin expression in the rat striatum, we found that the D2 antagonist haloperidol protects striatal neurons against expanded huntingtin-mediated toxicity. Two variant transcripts are generated by alternative splicing of the of D2 receptor gene, the D2R-Long and the D2R-Short, which are thought to play different functional roles. We show herein that overexpression of D2R-Short, but not D2R-Long in cell lines is associated with activation of the RhoA/ROCK signaling pathway. In striatal neurons in culture, the selective D2 agonist Quinpirole triggers phosphorylation of cofilin, a downstream effector of ROCK, which is abrogated by siRNAs that knockdown both D2R-Long and D2R-Short, but not by siRNAs targeting D2R-Long alone. Aggregate formation and neuronal death induced by expanded huntingtin, were potentiated by Quinpirole. This D2 agonist-mediated effect was selectively inhibited by the siRNA targeting both D2R-Long and D2R-Short but not D2R-Long alone. Our data provide evidence for a specific coupling of D2R-Short to the RhoA/ROCK/cofilin pathway, and its involvement in striatal vulnerability to expanded huntingtin. A new route for targeting Rho-ROCK signaling in Huntington's disease is unraveled with our findings.
\end{abstract}

\section{Introduction}

Huntington's disease (HD) is an inherited neurodegenerative disorder resulting from abnormal polyglutamine extension in the N-terminal region of the huntingtin protein (Exp-HTT; The Huntington's Disease Collaboration Group, 1993). It is characterized by chorea,

Correspondence: Jocelyne Caboche, ${ }^{8}$ Sorbonne Université, as above.

E-mail: jocelyne.caboche@upmc.fr

*S.B. and J.C. contributed equally to this work psychiatric disturbances, and cognitive impairments (Roze et al., 2008). Although Exp-HTT is expressed ubiquitously in the brain, a selective vulnerability of striatal neurons, which is causal in the neuropathology, is now well established in the disease. The dopaminergic hypothesis of this vulnerability arose during the past years. First, the striatum receives the densest dopaminergic (DA) innervation of the brain, and striatal neurodegeneration in HD is positively correlated with the gradient of DA in its rostro-caudal extension (Vonsattel et al., 1985). Second, in animal or cellular models, an exaggerated increase of DA produces spontaneous striatal death and 
HD-like behaviors (Luo et al., 1998; Cyr et al., 2003). Furthermore, D2 receptor (D2R) stimulation by DA acts in synergy with Exp-HTT to increase aggregate formation, neuritic retraction, mitochondrial dysfunctions, and striatal death (Charvin et al., 2005; Benchoua et al., 2008). In vivo, chronic treatment with the $\mathrm{D} 2 \mathrm{R}$ antagonist haloperidol protects against striatal dysfunctions induced by Exp-HTT in a lentiviral-based model system in rats (Charvin et al., 2008).

D2Rs belong to the structurally related G-protein-coupled receptors family, which are characterized by seven transmembrane segments (De Mei et al., 2009). D2Rs are classically identified as inhibitors of cyclic AMP production and activation of PKA via their coupling to Gai subunits (De Mei et al., 2009). D2Rs also affect many downstream effectors including $\mathrm{K}+$ channels (Greif et al., 1995), L-type $\mathrm{Ca}^{2+}$ channels and phospholipase C (PLC; Hernandez-Lopez et al., 2000), glutamate release (Bamford et al., 2004; Yin \& Lovinger, 2006), and intracellular signaling pathways including mitogen-activated protein kinases and Akt (Brami-Cherrier et al., 2002; reviewed in Vallone et al., 2000; Neve et al., 2004). We made the original observation that D2Rs were coupled to the Rho-ROCK signaling pathway, and that D2R-mediated activation of this pathway was responsible for a potentiation of ExpHTT-induced neuritic retraction and striatal neuron death (Deyts et al., 2009).

Two variant transcripts are generated by alternative splicing of the D2Rs gene and code for the D2R Long and D2R Short isoforms, respectively (De Mei et al., 2009). The D2R Short isoform differs from D2R Long by the deletion of 29 amino acids in the third intracellular loop of the receptor. These spliced variants are expressed in the same cell types and exhibit similar pharmacological characteristics with no isoform-specific pharmacological ligands identified so far. They possess a differential subcellular distribution, with D2R Short being preferentially presynaptic inhibitory autoreceptors and playing a preferential role in release modulation, (Lindgren et al., 2003) whereas D2R Long are predominantly postsynaptic receptors associated with G-protein-dependent and -independent intracellular signaling (De Mei et al., 2009). Because D2R-induced potentiation of ExpHtt-induced striatal phenotype (aggregate formation and striatal death) was totally blocked by selective inhibition or knockdown of Rho-ROCK signaling pathway (Deyts et al., 2009), we investigated herein a possible specific coupling of D2R Short or D2R Long to the Rho/ROCK signaling pathway and striatal vulnerability in HD.

\section{Materials and methods}

\section{HEK293 cell culture and transfection}

HEK293 cells were cultured and maintained in Dulbecco's modified Eagle's medium (DMEM; Invitrogen, Carlsbad, CA, USA) supplemented with $10 \%$ fetal bovine serum (FBS) and $0.1 \%$ penicillinstreptomycin. The day before transfection, the cells were plated in p100 mm dishes in DMEM without antibiotics. HEK293 cells were then transiently transfected using Lipofectamine (Invitrogen) with $4 \mu \mathrm{g}$ of plasmids encoding N-terminal YFP-tagged D2 receptor isoforms, YFP-D2R Long and YFP-D2R Short (generously provided by Dr Philippe Vernier (CNRS, France) or empty plasmid during $24 \mathrm{~h}$ later.

\section{Rho activity assay}

Rho activation was analyzed using Rho assay kit (Upstate Biotechnology, Waltham, CA, USA). After overnight starvation in $0.1 \%$ FBS, HEK293 cells expressing D2R Short or D2R Long isoforms of $\mathrm{D} 2 \mathrm{R}$ or the empty vector were stimulated with Quinpirole (10 $\mu \mathrm{M}, \mathrm{RBI})$ or Dopamine (DA; $100 \mu \mathrm{M}$, Calbiochem, San Diego, CA, USA) for $5 \mathrm{~min}$ at $37{ }^{\circ} \mathrm{C}$. Cells were then lysed in $10 \%$ glycerol, $50 \mathrm{~mm}$ Tris $\mathrm{pH} 7.2,1 \%$ Triton $\mathrm{X}-100,0.5 \%$ sodium deoxycholate, $0.1 \%$ SDS, $500 \mathrm{~mm} \mathrm{NaCl}, 10 \mathrm{~mm} \mathrm{MgCl}_{2}$, and complete protease inhibitor cocktail tablets (Roche, Indianapolis, IN, USA) for $15 \mathrm{~min}$ at $4{ }^{\circ} \mathrm{C}$ in a rotator. Homogenates were clarified by centrifugation at $13000 \mathrm{~g}$ at $4{ }^{\circ} \mathrm{C}$ for $5 \mathrm{~min}$. Rho activity was then measured according to the manufacturer's instructions. Rho-GTP was subjected to SDS-PAGE followed by western blotting with mouse anti-Rho (-A,-B,-C; 1/250; Millipore, Billerica, MA, USA). The bands were quantified by densitometric analysis using a dedicated macro application of SCION IMAGE software (Scion Corp., Frederick, MD, USA) and normalized to Total-Rho expression.

\section{RNA interference}

siRNAs were designed to interfere with the expression of both D2R isoforms (siRNA D2R Total: CCAGAGAGGACCCGGTAT) or only the D2R Long isoform (D2R Long siRNA: CCAGTGAACAGGCGGAGAA). Neurons were transfected at days in vitro (DIV4) with $50 \mathrm{~nm}$ of D2R Total siRNA, D2R Long-specific, or Control siRNA (Cont siRNA \#2, Ambion, Austin, TX, USA). The specificity of siRNAs was validated in primary striatal cultures $72 \mathrm{~h}$ after transfection. When indicated, primary neurons were transfected (DIV7) with either wild-type (HTT) or expanded-HTT (Exp-HTT; see methods below). Drug treatment and evaluation of aggregates or death were performed as indicated above.

\section{RNA isolation and RT-PCR}

RNA isolation and RT-PCR methods were performed as previously described (Deyts et al., 2009). The nucleotide sequences of the primers used to specifically amplify D2R Long and D2R Short receptors were as follows: D2R Long, forward 5'-AACTGTACC CACCCTGAGGA-3', reverse 5'-GTTGCTATGTAGACCGTG-3'; D2R Short, forward 5'-CACCACTCAAGGATGCTGCCCG-3', reverse 5'-GTTGCTATGTAGACCGTG-3'. ARP (Acidic Ribosomal Protein) was used as a housekeeping gene: ARP, forward $5^{\prime}$-GAA CGTCGTCTTCGTGTTCA-3', reverse 5'-AAAACCTGGACGAAG GAGGT-3'.

\section{Primary striatal culture}

Striatal neurons were dissected out from E14 embryos from pregnant Swiss mice (Janvier, France) as previously described (Charvin et al., 2005) and in accordance with the European Community guidelines on the Care and Use of Laboratory Animals (86/609/ EEC). Experiments were approved by the local ethic committee (reference 01435.02). For transfection assays, $0.8 \mu \mathrm{g}$ of GFPtagged plasmids encoding the first exon of human Huntingtin (HTT) containing either 25 or 103 continuous CAA or CAG repeats (HTT and Exp-HTT, respectively) was transfected at DIV 7, using Lipofectamine (Invitrogen). Three hours and a half after transfection, the medium was removed and replaced by fresh culture medium containing DA $(100 \mu \mathrm{M})$ or Quinpirole $(10 \mu \mathrm{M})$. Aggregate formation was evaluated $12 \mathrm{~h}$ after transfection by counting neurons expressing aggregated GFP vs. the total number of GFP-positive neurons. D2R agonist-induced potentiation of cell death was evaluated $24 \mathrm{~h}$ after transfection. Neurons containing condensed or fragmented nuclei, based on Hoechst labeling, were scored as dying cells. 


\section{Immunocytochemistry}

Striatal neurons were fixed in $2 \%$ paraformaldehyde in PBS for $40 \mathrm{~min}$ at room temperature (RT) and permeabilized with methanol/ acetone $(1: 1)$ for $10 \mathrm{~min}$ at $4{ }^{\circ} \mathrm{C}$. After 1-h incubation with blocking buffer (10\% normal goat serum in PBS, RT), cells were incubated overnight with primary antibodies in PBS at $4{ }^{\circ} \mathrm{C}$; mouse anti-MAP-2 (1/1000, Sigma Aldrich, St.Louis, MO, USA) or rabbit anti-P-cofilin (1/500, Cell Signaling, Technology, Ipswich, MA, USA). Cells were washed with PBS and incubated for $2 \mathrm{~h}$ at RT with corresponding Alexa-conjugated secondary antibodies (1/1000, Jackson Laboratories, Bar Harbor, Maine, USA). Nuclei were stained with Hoechst (1/20000 in PBS, Invitrogen). Coverslips were mounted using Mowiol (Calbiochem) and examined with a Leica DM4000B fluorescence microscope $(20 \times)$. In each experiment, conducted a minimum of three times, a total of at least 10 fields were randomly analyzed using IMAGEPRO-PLUS software.

\section{Statistical analysis}

Data are presented as mean \pm SEM. Statistics were performed using GRAPHPAD PRISM (GraphPad Software Inc., San Diego, CA). One-way or two-way ANova followed by post hoc Bonferroni's test were carried out for statistical analyses as indicated in the results. The difference between comparisons was considered to be significant when $P<0.05$.

\section{Results \\ Overexpression of D2R Short, but not D2R Long, activates the RhoA/ROCK signaling in HEK293 cells}

We previously showed that DA and the D2 agonist Quinpirole activate the RhoA/ROCK signaling pathway in primary cultures of striatal neurons (Deyts et al., 2009). In order to investigate a possible differential coupling of either D2R Short or D2R Long to the RhoA/ ROCK signaling pathway, we first used a heterologous cellular system cDNAs encoding these D2R-specific isoforms tagged to YFP, were transfected in HEK293 cells, and allowed expression of the receptors that were trafficked to the plasma membrane, as expected (Fig. 1A). We next measured Rho activity by measuring RhoA-GTP levels relative to Total-RhoA in transfected cells (Fig. 1B-C). After overnight serum deprivation, cells were treated with Quinpirole
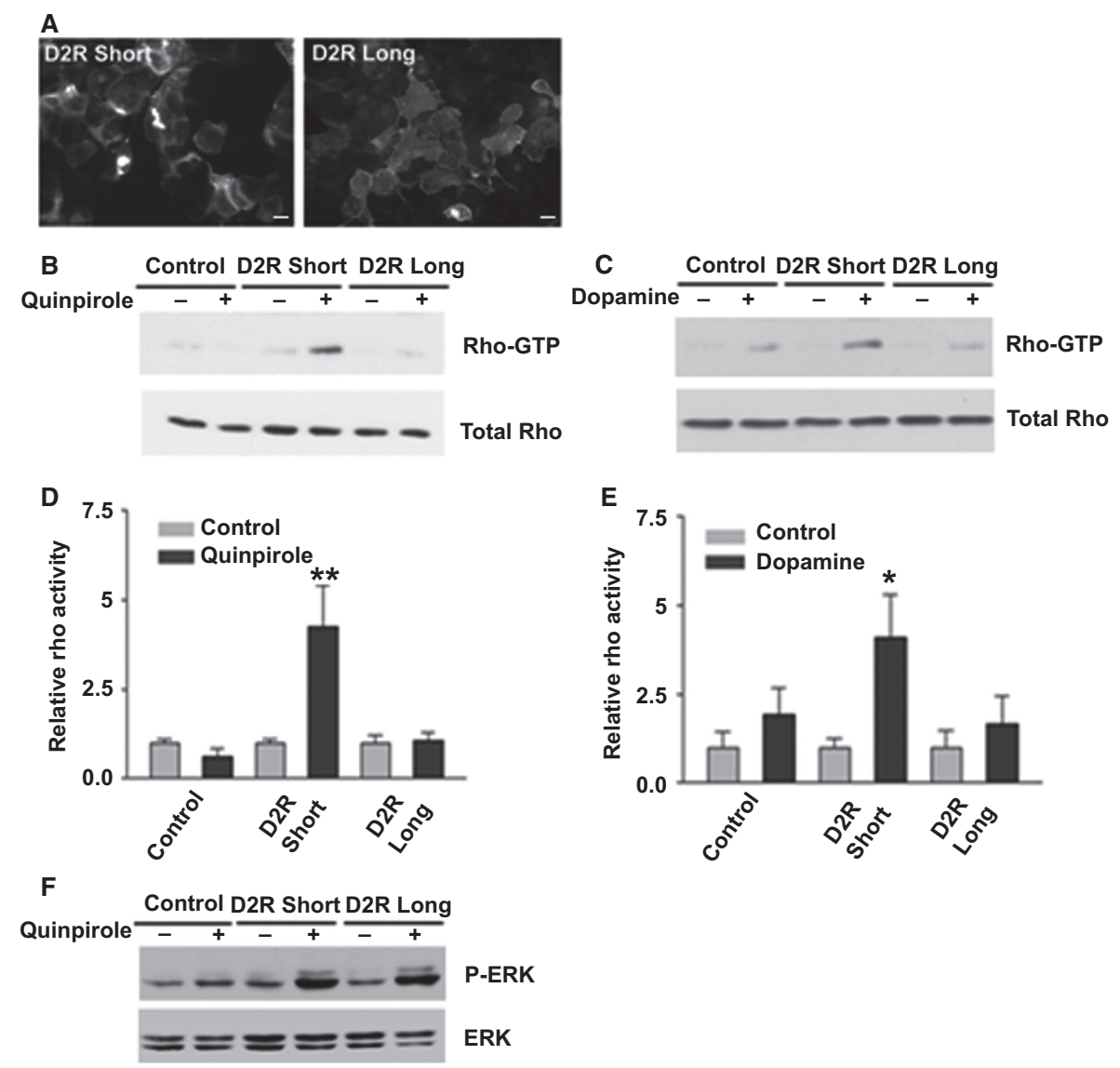

FIG. 1. Dopamine D2R Short specific coupling to the RhoA/ROCK signaling pathway in HEK293 cells. HEK 293 cells were transiently transfected with YFPtagged D2R Short and D2R Long (right panel) during 24 h. (A) Epifluorescence images showing the YFP-tagged D2R Short (left panel) and D2R Long (right panel) receptors expression in HEK293 cells. Note that both D2R Short and D2R Long are expressed at the plasma membrane. Scale bar: $10 \mu$ m. (B and C) RhoA-GTP pull-down assay with Rhotekin-GST and western blot detection of RhoA-GTP (up) and TOTAL-RhoA (down) were performed in D2R Short or D2R Long transfected cells after 5 min of Quinpirole $10 \mu \mathrm{M}(\mathrm{B})$ or $100 \mu \mathrm{M}$ DA (C) treatment. Control lanes correspond to cells transfected with an empty vector. (D and E) Quantification of RhoA-GTP activity normalized to TOTAL-RhoA protein was performed by densitometric analysis from three independent experiments after Quinpirole (D) or Dopamine (E) treatment. (D-E) Two-way ANovA followed by Bonferroni's post hoc analysis $(n=3$ independent experiments; $* P<0.05, * * P<0.01$ compared with non-treated cells. (F) Quinpirole-induced ERK phosphorylation in D2R Short- and D2R Long-expressing cells by comparison with YFP-alone transfected cells (Control). Quinpirole treatment (10 $\mu \mathrm{M}, 5 \mathrm{~min})$ was able to induce ERK phosphorylation in D2R Long and D2R Short receptors-expressing cells. No changes in total ERK protein were detected. 
(10 $\mu \mathrm{M}$; Fig. 1B) or Dopamine (DA; $100 \mu \mathrm{M}$; Fig. 1C) for $5 \mathrm{~min}$. RhoA was activated by Quinpirole in D2R Short-expressing cells ( $P=0.0028$ between Control and Quinpirole-treated cells) but not D2R Long-expressing cells $(P>0.9999$ non-significant between Control and Quinpirole-treated cells; Fig. 1B and D). Similar results were obtained in DA-treated cells $(P=0.0292$ between D2R Shortexpressing cells treated or not with Quinpirole; Fig. 1C and E). In order to verify the functionality of the transfected D2Rs, ERK activation by Quinpirole was studied. As expected, a robust induction of ERKs phosphorylation was observed in response to Quinpirole in both D2R Short and D2R Long transfected cells (Fig. 1F). These results clearly demonstrate a specific coupling of D2R Short but not D2R Long to RhoA activation in HEK293 cells.

\section{siRNAs strategy for knocking down D2 receptors in striatal neurons in culture}

We next designed a siRNA-based strategy aimed at silencing D2Rs expression in striatal neurons in culture. As a first step, we analyzed endogenous expression levels of mRNAs encoding D2R Short and D2R Long, in primary striatal neurons in culture (DIV 7 and DIV 15; Fig. 2A). To amplify the D2R Long isoform, we used a forward primer annealing the exon 6, which is lost in D2R Short. To amplify D2R Short, the forward primer was designed in order to specifically bind to the spliced junction between exons 5 and 7 . Reverse primers were designed to anneal with exon 7, common to both isoforms. RT-PCR showed that mRNAs encoding D2R Short and D2R Long were expressed in striatal neurons in culture (Fig. 2A). Then we designed siRNAs in order to knockdown D2Rs (Fig. 2B). D2R Total siRNA corresponded to a sequence of exon 7 common to both D2R Short and D2R Long mRNA sequences. The siRNA target sequence for specific knockdown of D2R Long mRNA was located in the exon 6, and thus absent in the D2R Short splice variant. Validation of these two siRNAs (D2R Total and D2R Long) was achieved after transfection in striatal neurons. As expected, semiquantitative RT-PCR showed that D2R Long siRNA knocked down D2R Long but not D2R Short expression (Fig. 2C), while D2R Total siRNA decreased expression of both isoforms (Fig. 2D).
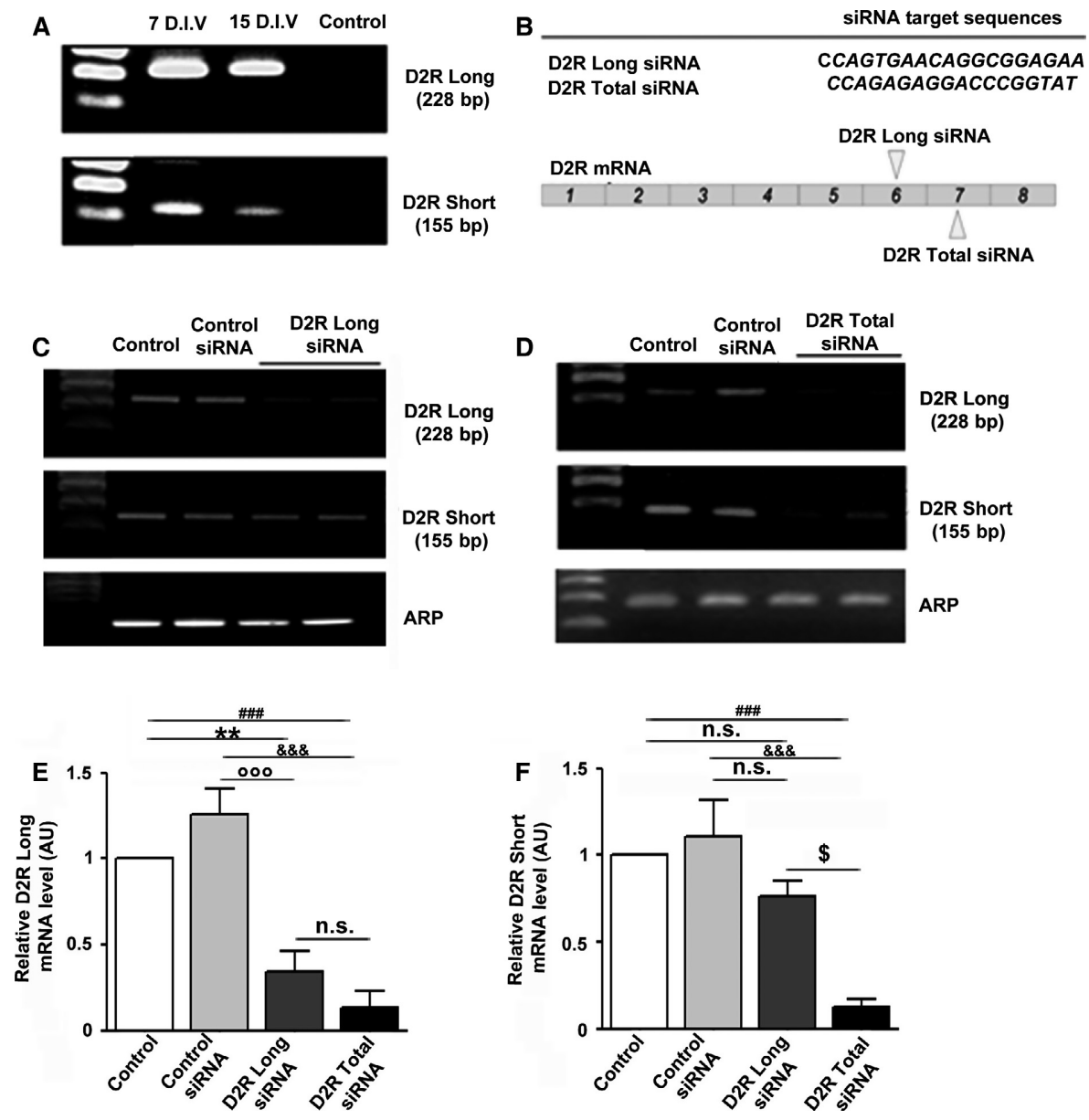

FIG. 2. D2R Long and D2R Short expression levels in primary striatal neurons and validation of D2 receptors specific siRNAs. (A) RT-PCR with specific primers showed that D2S and D2L mRNAs are expressed in E14 striatal neurons in culture at DIV7 and DIV15. (B) siRNA target sequences and designed strategy used to knockdown both isoforms of D2 receptors (D2R Total siRNA) or specifically the Long isoform (D2R Long siRNA). (C-D) Validation by semiquantitative RT-PCR of D2R Long (C) and D2R Total (D) siRNA efficacy. Lower panels show mRNA levels of the housekeeping gene ARP (acidic ribosomal protein). (E-F) Quantification of D2L (E) and D2S (F) mRNA levels after siRNA transfections. Reported are the ratios of D2R over ARP mRNAs. As expected, D2L but not D2S mRNA expression is abolished by D2R Long siRNA (C) compare upper and middle panels; (E-F) compare Control and D2R Long siRNA). Both D2R Short and D2R Long mRNAs are down-regulated by D2R Total siRNA (D) compare upper and middle panels, (E-F) compare Control and D2R total siRNA). (E-F) Data are expressed as mean \pm SEM of 3-4 independent experiments. Statistical analyses were performed using one-way ANova followed by Bonferroni's post hoc analysis. n.s., non-significant; $* * P<0.005$ when comparing Control with D2R Long siRNA, \#\#\# $P<0.001$ when comparing Control with D2R total siRNA, ${ }^{\circ \circ} P<0.001$ when comparing Control siRNA with D2R Long siRNA, \&\&\& $P<0.001$ when comparing Control siRNA with D2R total siRNA, ${ }^{\$} P<0.05$ when comparing D2R Long siRNA with D2R Total siRNA. 

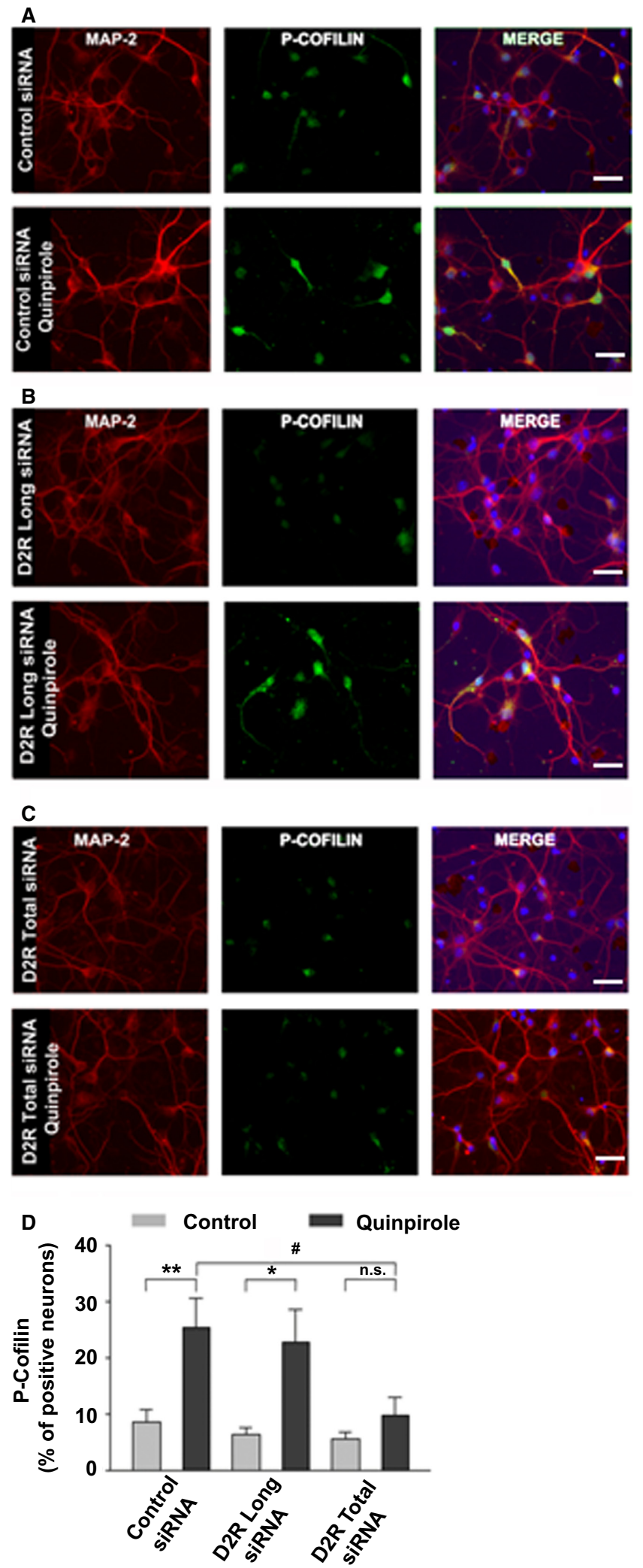

FIG. 3. Comparative effect of D2R Long siRNA and D2R Total siRNA on Quinpirole-induced phosphorylation of cofilin in cultured striatal neurons. (A-C) Representative immunofluorescence images showing MAP2 (red) and P-cofilin (green) immunoreactivity in control conditions (upper panels) or 20 min after Quinpirole treatments (10 $\mu \mathrm{m}$, lower panels) in striatal neurons transfected with Control siRNA (A), D2R Long siRNA (B) and D2R Total siRNA (C). Nuclei were labeled using Hoechst (blue, merge panels). (A-C) Scale bar: $30 \mu \mathrm{m}$. (D) Phospho-cofilin positive neurons were calculated as a percentage relative to the total number of neurons identified by Hoechst staining. Data are expressed as mean \pm SEM of three independent experiments at least. Statistics: two-way ANova followed by Bonferroni post hoc analysis $* P<0.05$, $* * P<0,01$ when comparing Control and Quinpirole-treated cells, \# when comparing cells transfected with Control siRNA with D2R Total siRNA. n.s.,Non-significant. 
Quantifications showed that both D2R Long and D2R Total siRNAs decreased D2R Long mRNA levels when compared to Control (66\%, $P=0.042$ and $86.8 \%, P=0.0003$, respectively; Fig. 2E), while D2R Short mRNA levels were significantly decreased after D2R Total siRNA treatment only $(P=0.0005$; Fig. $2 \mathrm{~F})$. We designed an siRNA targeting the spliced junction betweens exons 5 and 7, which failed to be efficient for the knowkdown of D2R Short (data not shown). Therefore, in the following experiments, we compared the effects of D2R Long and D2R Total siRNAs on D2R agonist and DA-induced RhoA/ROCK signaling in striatal neurons.

\section{Comparative role of D2R Long siRNA and D2R Total siRNA in}

Quinpirole-induced phosphorylation of cofilin in striatal neurons

We previously showed that Quinpirole induces phosphorylation of cofilin, a downstream target of RhoA/ROCK signaling, and this phosphorylation event was totally reversed by ROCK inhibitors and siRNA against ROCKII (Deyts et al., 2009). Thus, phosphorylated cofilin serves as an excellent index of ROCK activation to study D2Rs coupling to RhoA/ROCK pathway in striatal neurons.

Striatal neurons were transfected at DIV4 for $72 \mathrm{~h}$ with D2R Total siRNA or D2R Long siRNA and stimulated or not with Quinpirole (10 $\mu \mathrm{M}, 20 \mathrm{~min}$ ) or DA (not shown). The phosphorylation of cofilin was analyzed by immunocytochemical detection owing to a phospho-specific antibody. When transfected with the Control siRNA, striatal neurons showed a significant increase in phosphocofilin immunoreactivity after Quinpirole treatment $(25 \%$ vs. $9 \%$ in untreated neurons; $P=0.001$; Fig. $3 \mathrm{~A}$ and $\mathrm{D}$ ). In striatal neurons transfected with D2R Long siRNA, Quinpirole induced a similar increase in phospho-cofilin immunoreactivity $(23 \% ; P=0.0117$ between Control and Quinpirole-treated cells; Fig. 3B and D). However, knocking down both D2Rs isoforms using D2R Total siRNA completely blocked Quinpirole-induced cofilin phosphorylation ( $P=0.0152$ between Quinpirole-treated neurons expressing Control siRNA and D2R Total siRNA; Fig. 3C and D). Altogether these results support that stimulation of D2R Short, but not D2R Long, is coupled to phosphorylation of cofilin in striatal neurons.

\section{Comparative role of D2R Long siRNA and D2R Total siRNA on Quinpirole-induced potentiation of Exp-HTT toxicity and aggregate formation}

Using primary cultures of striatal neurons expressing exon 1 of HTT with a polyglutamine stretch (103Q: Exp-HTT) fused to EGFP, we previously showed that DA, via D2R stimulation, potentiates aggregate formation and striatal death induced by Exp-HTT (Charvin et al., 2005). The RhoA/ROCK signaling pathway is critical for D2R-mediated effects on aggregate formation and neuronal death (Deyts et al., 2009). We thus wished to compare the relative contribution of D2R Long and D2R Short in these effects. In striatal neurons transiently transfected with Exp-HTT, aggregate formation can be easily detectable owing to GFP Tag (Fig. 4A). In neurons co-transfected with Exp-HTT together with the Control siRNA, a treatment with $10 \mu \mathrm{M}$ of Quinpirole increased the percentage of transfected neurons containing aggregates $(+36 \%$; Fig. 4B) and the D2R Long siRNA did not prevent this enhanced aggregation (Fig. 4B; $P>0.9999$ non-significant between Control siRNA and D2R Long siRNA-treated cells). By contrast, the D2R Total siRNA reversed the potentiation of aggregate formation induced by Quinpirole (Fig. 4B; $P=0.0203$ ), thus supporting a specific role of D2R Short on Quinpirole-mediated aggregation of Exp-HTT.
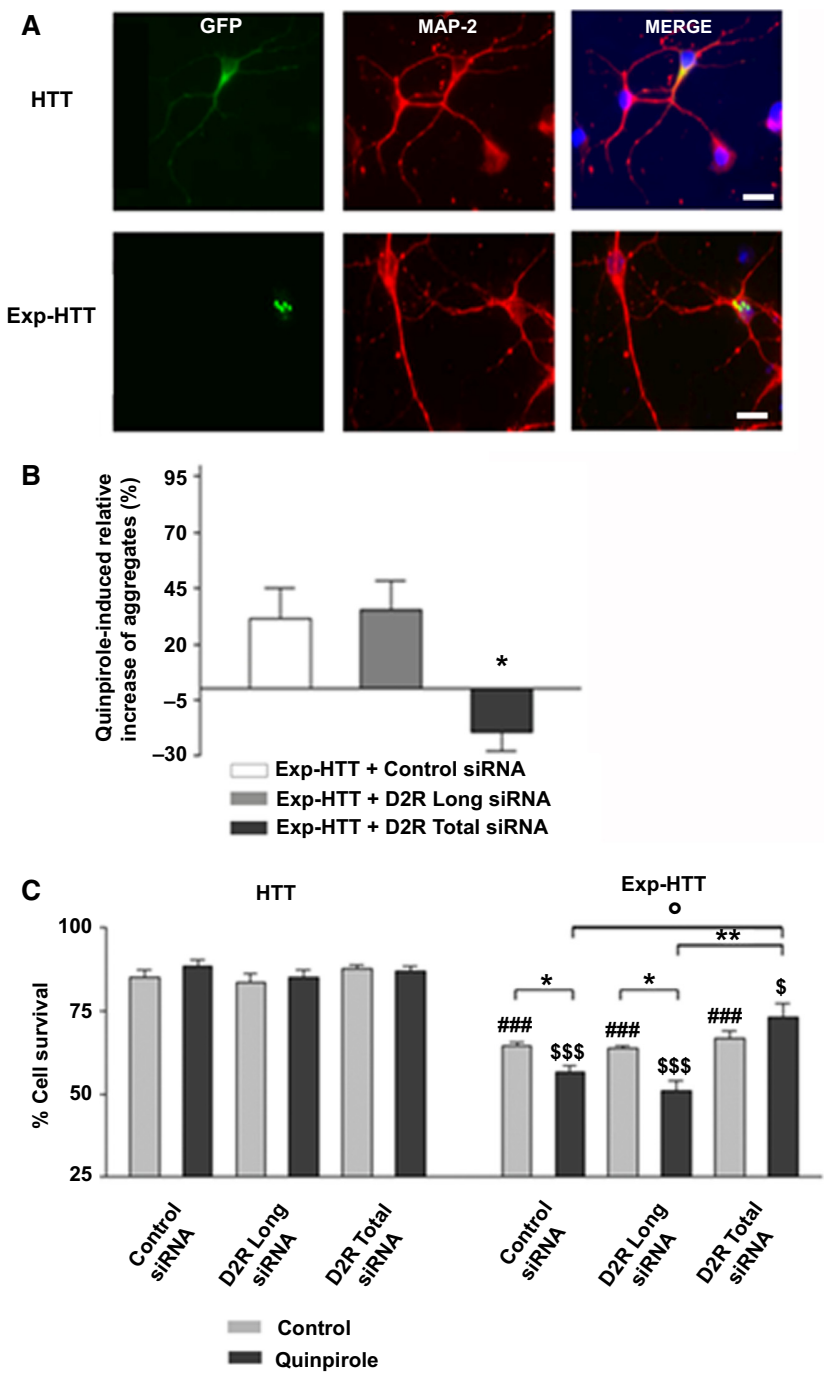

FIG. 4. Comparative effect of D2R Long siRNA and D2R Total siRNA on Quinpirole-induced potentiation of Exp-HTT toxicity and aggregate formation. (A) GFP-tagged Huntingtin (HTT) constructs (green) showing the expression pattern of diffused wild-type HTT (25Q-HTT) and aggregated Exp-HTT (103Q-HTT) in transfected striatal neurons (MAP2, red). (A) Scale bar: $20 \mu \mathrm{m}$ (B). Quantification of Quinpirole $(10 \mu \mathrm{M})$-induced increase of aggregates in striatal neurons co-transfected with Exp-HTT and siRNAs (Control, D2R Long or D2R Total). (C) The percentage of cell survival in striatal neurons co-transfected with siRNAs (Control, D2R Long, or D2R Total) and HTT (left panel) or Exp-HTT (right panel) was measured $24 \mathrm{~h}$ after Quinpirole $(10 \mu \mathrm{M})$ based on Hoechst labeling. Data are expressed as mean \pm SEM from three independent experiments (100 transfected neurons per condition and experiment). Statistical analyses were performed using two-way ANOva followed by Bonferroni's post hoc analysis. ${ }^{\# \# \#} P<0.001$ when comparing untreated HTT and Exp-HTT-expressing cells. ${ }^{\$} P<0.05$; ${ }^{\$}$ ${ }^{\$} P<0.001$ when comparing Quinpirole-treated HTT- and Exp-HTT-expressing cells. ${ }^{*} P<0.05$, ${ }^{*} P<<0.005$, when comparing Exp-HTT-expressing cells treated or not with Quinpirole. ${ }^{\circ} P<0.05$, when comparing Quinpiroletreated Exp-HTT-expressing cells in the presence of Control siRNA and D2R Total siRNA.

We next studied striatal death induced by Exp-HTT alone or in the presence of Quinpirole. Exp-HTT produced a significant striatal death $(P=0.0033$ between untreated HTT and Exp-HTT-expressing cells), which was potentiated by Quinpirole $(P=0.0444$ between Exp-HTT-expressing cells treated or not with Quinpirole; Fig. 4C). D2R Long siRNA did not significantly affect the ratio of striatal death induced by ExpHTT in the presence of Quinpirole 

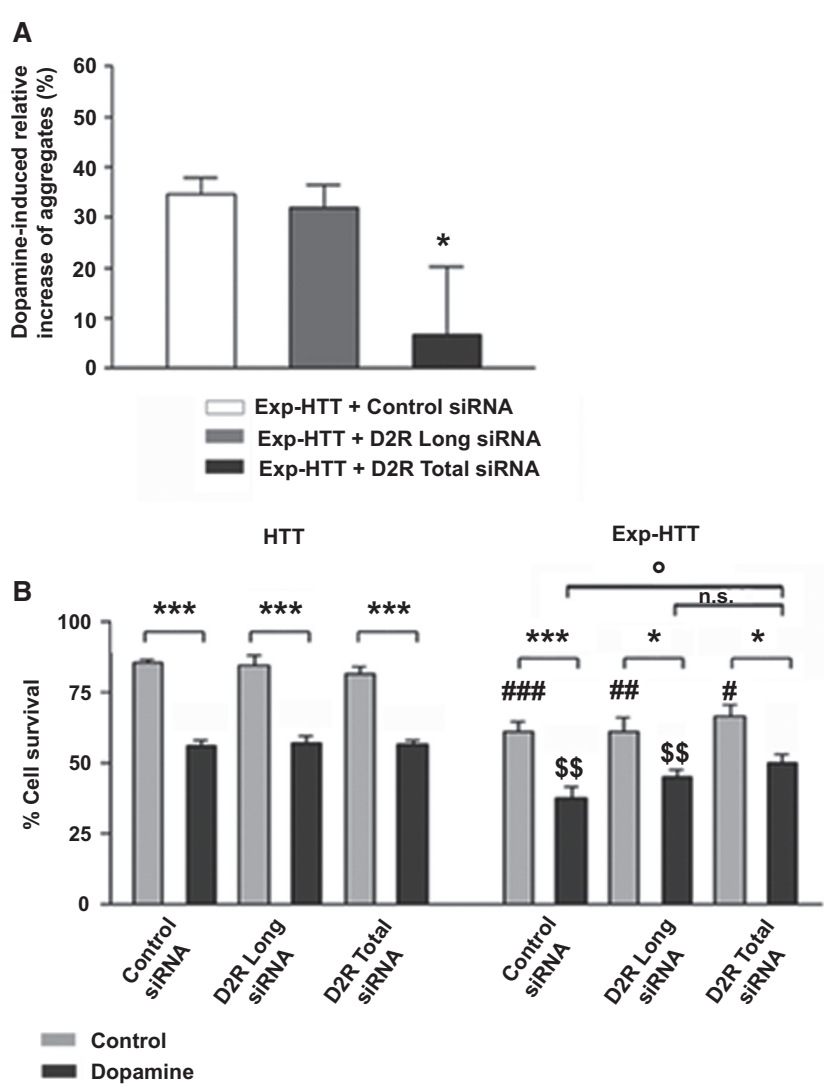

FIG. 5. DA-induced potentiation of Exp-HTT mediated toxicity is linked to D2R Short stimulation in striatal neurons. GFP-tagged Huntingtin (HTT) constructs of wild-type HTT (25Q-HTT) and Exp-HTT (103Q-HTT) were transfected in striatal neurons. (A) Quantification of DA-induced increase of aggregates in striatal neurons co-transfected with Exp-HTT and siRNAs (Control, D2R Long, or D2R Total; DA $100 \mu \mathrm{M}$ ). (B) Quantifications of the percentage of cell survival in striatal neurons co-transfected with siRNAs (Control, D2R Long, or D2R Total) and HTT (left panel) or Exp-HTT (right panel) was measured $24 \mathrm{~h}$ after DA $(100 \mu \mathrm{M})$ based on Hoechst labeling. Data are expressed as mean \pm SEM from three independent experiments (100 transfected neurons per condition and experiment). Statistical analyses were performed using two-way ANOVA followed by Bonferroni's post hoc analysis. ${ }^{\# \# \# P} P<0.001 ;{ }^{\# \#} P<0.005 ;{ }^{\#} P<0.05$ when comparing untreated HTT and Exp-HTT-expressing cells. ${ }^{\$} P<0.005$ when comparing DA-treated HTT- and Exp-HTT-expressing cells. ${ }^{*} P<0.05$, ${ }^{* * *} P<0.001$, when comparing Exp-HTT-expressing cells treated or not with DA. ${ }^{\circ} P<0.05$, when comparing DA-treated Exp-HTT-expressing cells in the presence of Control siRNA and D2R Total siRNA.

( $P>0.9999$ non-significant; Fig. 4C). By contrast, D2R Total siRNA completely reversed Quinpirole-induced toxicity in ExpHTT-expressing neurons $(P>0.9999$ non-significant between D2R Total siRNA cells treated or not with Quinpirole), thus supporting a preferential role of D2R-Short in this effect.

Comparative role effect of D2R Long siRNA and D2R Total siRNA on DA-induced potentiation of Exp-HTT aggregate formation and death

When applied at high doses $(500 \mu \mathrm{M})$, DA induces striatal death through a mechanism involving the formation of reactive oxygen species (ROS) formation and the activation of pro-apoptotic pathways (Luo et al., 1998). This effect is accentuated with lower doses of DA $(100 \mu \mathrm{M})$ in Exp-HTT-expressing cells (Charvin et al., 2005), due to a dual role on ROS production (independent of DA

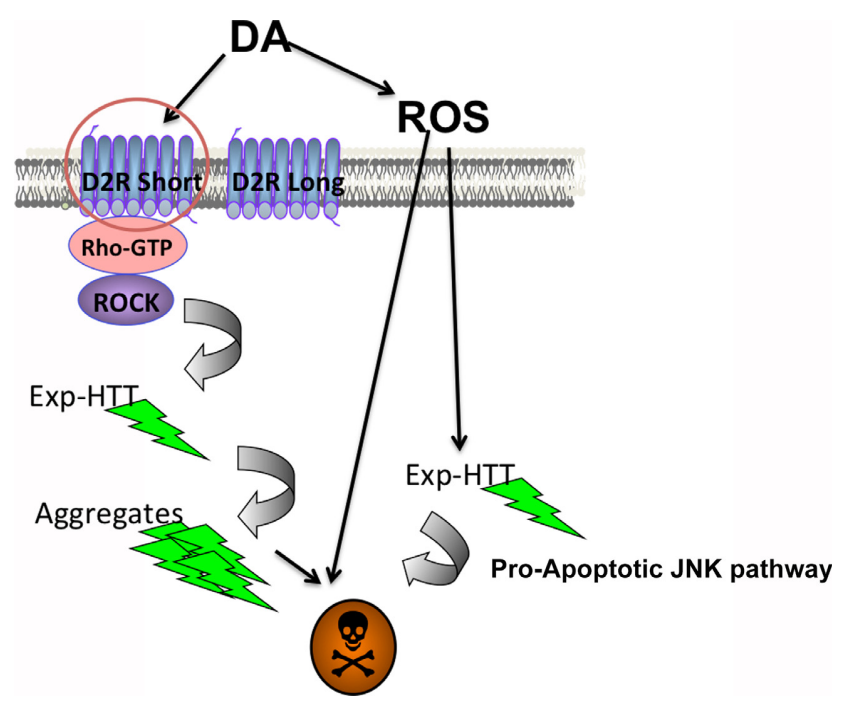

FIG. 6. Schematic representation of DA-mediated toxicity and striatal vulnerability in HD. During aging, DA auto-oxidation potentiates pro-apoptotic pathways that are activated by Exp-HTT (Charvin et al., 2005; Garcia et al., 2004). By acting on D2R Short isoform, DA also activates the Rho/ROCK pathway, which potentiates Exp-HTT aggregate formation and neuronal death, two features of the pathogenesis of the disease. As a result, DA shows a dual implication in Exp-HTT-mediated toxicity.

receptors stimulation) and $\mathrm{D} 2 \mathrm{R}$ stimulation. We analyzed the selective role of D2R Long siRNA and D2R Total siRNA in these effects. In Exp-HTT-expressing neurons, DA produced a significant increase in aggregate formation, which was not affected by $\mathrm{D} 2 \mathrm{R}$ Long siRNA $(P>0.9999$ non-significant between Control siRNA and D2R Long siRNA) but was completely abolished by D2R Total siRNA $(P=0.0106$ between Control siRNA and D2R Total siRNA; Fig. 5A). These data strongly suggest a role of D2R Short in DAmediated aggregate potentiation.

DA-induced striatal death was significantly higher in neurons overexpressing Exp-HTT than the wild-type HTT $(P=0.0035$ between DA-treated HTT-expressing cells and DA-treated ExpHTT-expressing cells; Fig. 5B), thus illustrating the combined toxicity of DA and Exp-HTT. As previously shown, the total knockdown of D2R partially but significantly reversed this effect in Exp-HTTexpressing cells $(P=0.0419)$. The effect of DA was similar in DAtreated HTT and Exp-HTT-expressing cells in the presence of D2R Total siRNA $(P>0.9999$ non-significant). Of interest, the toxicity induced by DA in Exp-HTT-expressing cells was not modified by D2R Long siRNA $(P=0.0144)$, data that confirm a specific role of D2R Short in DA-mediated toxicity (Fig. 5B).

\section{Discussion}

In striatal neurons, we previously showed a coupling between D2R and the RhoA/ROCK signaling pathway (Deyts et al., 2009). Here we extend these observations and provide evidence for a specific coupling of D2R Short to this pathway, which affects striatal vulnerability to Exp-HTT.

Rho-associated kinases (ROCKs) are Ser/Thr protein kinases and downstream effectors of the small GTPases RhoA. They are important regulators of cell growth, migration, and apoptosis via the control of actin cytoskeletal assembly (for review Julian \& Olson, 2014). In the context of HD, it has been shown that inhibitors of ROCK decrease Exp-HTT aggregation formation (Burnett et al., 2008; Bauer et al., 2009, 2012) and activates the proteasome 
degradation pathways (Bauer et al., 2009). This pathway is significantly enriched in large-scale HTT-interacting protein networks and has been proposed as a modifier of Exp-HTT (Shirasaki et al., 2012; Tourette et al., 2014). Rho/ROCK inhibition using pharmacological or molecular approaches reduces the aggregation and toxicity of proteins with expanded glutamine including HTT (Bauer et al., 2009, 2012), androgen receptor in drosophila, or cell line models (Pollitt et al., 2003). Inhibition of Rho/ROCK pathway also shows benefits in Alzheimer disease (Hahmann \& Schroeter, 2010; Li et al., 2013) as well as in other neurodegenerative diseases (for review Hensel et al., 2015). Cofilin is a well-known substrate of Rho/ROCK pathway and is required for the actin cytoskeleton dynamics and reorganization of actin filaments (Bamburg \& Wiggan, 2002). Cofilin phosphorylation participates in neuronal dystrophy induced by beta-amyloid in Alzheimer's disease pathology (Heredia et al., 2006). In striatal neurons in culture, the inhibition of ROCK reversed D2R-mediated phosphorylation of cofilin, along with Exp-HTT-induced aggregate formation, neuritic retraction, and neuronal death (Deyts et al., 2009). Importantly, we show here that the coupling of D2R to RhoA/ROCK is specific of the Short, spliced version of D2R.

The molecular mechanisms at the origin of the D2R Shortmediated activation of Rho remain to be established. One candidate could be the scaffolding protein $\beta$-arrestin2, which facilitates some facets of D2R signaling, in addition to its canonical role in receptor internalization (Beaulieu et al., 2005). Specific role of $\beta$-Arrestin2 in D2R Short, but not D2R Long-mediated ERK activation, has been described (Kim et al., 2004). On the other hand, $\beta$-arrestins, which interact with the exchange factor for RhoGTPases PDZ-RhoGEF (Oleksy et al., 2006), are associated with the activation of Rho downstream the Angiotensin A2 receptors (Barnes et al., 2005).

The first neuronal population affected in HD is the D2R expressing medium spiny neurons (MSNs; Roze et al., 2010). In mice and human, neuropil aggregates appear first in the globus pallidus and the substantia nigra, two brain regions where D2R are enriched (Albin et al., 1992; Li et al., 2001). Locally they are thought to be toxic by poisoning the axonal transport (Li et al., 2001; Lee et al., 2004). Based on the present work, we propose that Exp-HTTinduced toxicity is exaggerated by DA via two routes (see Fig. 6): one involves auto-oxidation of DA (Luo et al., 1998) and potentiation of pro-apoptotic pathways that are activated by Exp-HTT (Garcia et al., 2004; Charvin et al., 2005). The other one is associated with the stimulation of D2R Short isoform, which is coupled to the activation of the Rho-ROCK signaling pathway, the formation of aggregates, and neuronal death.

In addition to its important role in striatal vulnerability in HD, alterations of D2R-dependent signaling have been implicated in other brain pathologies such as schizophrenia, attention deficit hyperactivity disorder, Tourette syndrome, obsessive-compulsive disorder, Parkinson's disease, and drug addiction (Iversen \& Iversen, 2007; Seeman, 2010). The identification of D2Rs isoforms involved in these pathologies is of crucial importance for the development of new therapy. Herein, we provide evidence that interfering with D2R Short coupling to Rho might constitute a new and promising therapeutic strategy in HD and probably other brain pathologies.

\section{Acknowledgements}

We gratefully acknowledge Dr Peter Vanhoutte and Dr Nicolas Heck (UMR CNRS-8246 CNRS et UMRS-INSERM 1130 Université Pierre et Marie Curie-Paris) for their fruitful comments on the manuscript report. BGR was a recipient from Ministerio de Ciencia e Innovación, Programa Nacional de
Movilidad de Recursos Humanos, Plan nacional I-D+I 2008-2011 (Spain). Supports for JC was from Centre National pour la Recherche Scientifique, Institut National de la Santé et de la Recherche Médicale and Université Pierre et Marie Curie-Paris 6.

\section{Abbreviations}

D2R, D2 receptors; D2R Long, D2 receptor Long isoform; D2R Short, D2 receptor Short isoform; DA, Dopamine; Exp-HTT, Expanded Huntingtin; HD, Huntington's disease; HTT, Huntingtin.

\section{References}

Albin, R.L., Reiner, A., Anderson, K.D., Dure, L.S., Handelin, B., Balfour, R., Whetsell, W.O. Jr, Penney, J.B. et al. (1992) Preferential loss of striato-external pallidal projection neurons in presymptomatic Huntington's disease. Ann. Neurol., 31, 425-430.

Bamburg, J.R. \& Wiggan, O.P. (2002) ADF/cofilin and actin dynamics in disease. Trends Cell Biol., 12, 598-605.

Bamford, N.S., Robinson, S., Palmiter, R.D., Joyce, J.A., Moore, C. \& Meshul, C.K. (2004) Dopamine modulates release from corticostriatal terminals. J. Neurosci., 24, 9541-9552.

Barnes, W.G., Reiter, E., Violin, J.D., Ren, X.R., Milligan, G. \& Lefkowitz, R.J. (2005) beta-Arrestin 1 and Galphaq/11 coordinately activate RhoA and stress fiber formation following receptor stimulation. J. Biol. Chem., 280, 8041-8050.

Bauer, P.O., Wong, H.K., Oyama, F., Goswami, A., Okuno, M., Kino, Y., Miyazaki, H. \& Nukina, N. (2009) Inhibition of Rho kinases enhances the degradation of mutant huntingtin. J. Biol. Chem., 284, 13153-13164.

Bauer, P.O., Hudec, R., Goswami, A., Kurosawa, M., Matsumoto, G., Mikoshiba, K. \& Nukina, N. (2012) ROCK-phosphorylated vimentin modifies mutant huntingtin aggregation via sequestration of IRBIT. Mol. Neurodegener., 7, 43 .

Beaulieu, J.M., Sotnikova, T.D., Marion, S., Lefkowitz, R.J., Gainetdinov, R.R. \& Caron, M.G. (2005) An Akt/beta-arrestin 2/PP2A signaling complex mediates dopaminergic neurotransmission and behaviour. Cell, 122, 261-273.

Benchoua, A., Trioulier, Y., Diguet, E., Malgorn, C., Gaillard, M.C., Dufour, N., Elalouf, J.M., Krajewski, S. et al. (2008) Dopamine determines the vulnerability of striatal neurons to the N-terminal fragment of mutant huntingtin through the regulation of mitochondrial complex II. Hum. Mol. Genet., 17, 1446-1456.

Brami-Cherrier, K., Valjent, E., Garcia, M., Pagès, C., Hipskind, R.A. \& Caboche, J. (2002) Dopamine induces a PI3-kinase-independent activation of Akt in striatal neurons: a new route to cAMP response element-binding protein phosphorylation. J. Neurosci., 22, 8911-8921.

Burnett, B.G., Andrews, J., Ranganathan, S., Fischbeck, K.H. \& Di Prospero, N.A. (2008) Expression of expanded polyglutamine targets profilin for degradation and alters actin dynamics. Neurobiol. Dis., 30, 365-374.

Charvin, D., Vanhoutte, P., Pages, C., Borrelli, E. \& Caboche, J. (2005) Unraveling a role for dopamine in Huntington's disease: the dual role of reactive oxygen species and D2 receptor stimulation. Proc. Natl. Acad. Sci. USA, 102, 12218-12223.

Charvin, D., Roze, E., Perrin, V., Deyts, C., Betuing, S., Pages, C., Regulier, E., Luthi-Carter, R. et al. (2008) Haloperidol protects striatal neurons from dysfunction induced by mutated huntingtin in vivo. Neurobiol. Dis., 29, 22-29.

Cyr, M., Beaulieu, J.M., Laakso, A., Sotnikova, T.D., Yao, W.D., Bohn, L.M., Gainetdinov, R.R. \& Caron, M.G. (2003) Sustained elevation of extracellular dopamine causes motor dysfunction and selective degeneration of striatal GABAergic neurons. Proc. Natl. Acad. Sci. USA, 100, 11035-11040.

De Mei, C., Ramos, M., Iitaka, C. \& Borrelli, E. (2009) Getting specialized: presynaptic and postsynaptic dopamine D2 receptors. Curr. Opin. Pharmacol., 9, 53-58.

Deyts, C., Galan-Rodriguez, B., Martin, E., Bouveyron, N., Roze, E., Charvin, D., Caboche, J. \& Betuing, S. (2009) Dopamine D2 receptor stimulation potentiates PolyQ-Huntingtin-induced mouse striatal neuron dysfunctions via Rho/ROCK-II activation. PLoS One, 4, e8287.

Garcia, M., Charvin, D. \& Caboche, J. (2004) Expanded Huntingtin activates the c-JunN terminal kinase/c-Jun pathway prior to aggregate formation in striatal neurons in culture. Neuroscience, 127, 859-870.

Greif, G.J., Lin, Y.J., Liu, J.C. \& Freedman, J.E. (1995) Dopamine-modulated potassium channels on rat striatal neurons: specific activation and cellular expression. J. Neurosci., 15, 4533-4544. 
Hahmann, C. \& Schroeter, T. (2010) Rho-kinase inhibitors as therapeutics: from pan inhibition to isoform selectivity. Cell. Mol. Life Sci., 67, 171-177.

Hensel, N., Rademacher, S. \& Claus, P. (2015) Chatting with the neighbors: crosstalk between Rho-kinase (ROCK) and other signaling pathways for treatment of neurological disorders. Front. Neurosci., 9, 198.

Heredia, L., Helguera, P., de Olmos, S., Kedikian, G., Sola Vigo, F., LaFerla, F., Staufenbiel, M., de Olmos, J. et al. (2006) Phosphorylation of actin-depolymerizing factor/cofilin by LIM-kinase mediates amyloid betainduced degeneration: a potential mechanism of neuronal dystrophy in Alzheimer's disease. J. Neurosci., 26, 6533-6542.

Hernandez-Lopez, S., Tkatch, T., Perez-Garci, E., Galarraga, E., Bargas, J., Hamm, H. \& Surmeier, D.J. (2000) D2 dopamine receptors in striatal medium spiny neurons reduce L-type $\mathrm{Ca} 2+$ currents and excitability via a novel PLC[beta]1-IP3-calcineurin-signaling cascade. J. Neurosci., 20, 8987-8995.

Iversen, S.D. \& Iversen, L.L. (2007) Dopamine: 50 years in perspective. Trends Neurosci., 30, 188-193.

Julian, L. \& Olson, M.F. (2014) Rho-associated coiled-coil containing kinases (ROCK). Small GTPases, 5, e29846 1-12.

Kim, S.J., Kim, M.Y., Lee, E.J., Ahn, Y.S. \& Baik, J.H. (2004) Distinct regulation of internalization and mitogen-activated protein kinase activation by two isoforms of the dopamine D2 receptor. Mol. Endocrinol., 18, 640652.

Lee, W.C., Yoshihara, M. \& Littleton, J.T. (2004) Cytoplasmic aggregates trap polyglutamine-containing proteins and block axonal transport in a Drosophila model of Huntington's disease. Proc. Natl. Acad. Sci. USA, 101, 3224-3229.

Li, H., Li, S.H., Yu, Z.X., Shelbourne, P. \& Li, X.J. (2001) Huntingtin aggregate-associated axonal degeneration is an early pathological event in Huntington's disease mice. J. Neurosci., 21, 8473-8481.

Li, M., Yasumura, D., Ma, A.A., Matthes, M.T., Yang, H., Nielson, G., Huang, Y., Szoka, F.C. et al. (2013) Intravitreal administration of HA1077, a ROCK inhibitor, improves retinal function in a mouse model of huntington disease. PLoS One, 8, e56026.

Lindgren, N., Usiello, A., Goiny, M., Haycock, J., Erbs, E., Greengard, P., Hokfelt, T., Borrelli, E. et al. (2003) Distinct roles of dopamine D2L and D2S receptor isoforms in the regulation of protein phosphorylation at presynaptic and postsynaptic sites. Proc. Natl. Acad. Sci. USA, 100, 4305-4309.
Luo, Y., Umegaki, H., Wang, X., Abe, R. \& Roth, G.S. (1998) Dopamine induces apoptosis through an oxidation-involved SAPK/JNK activation pathway. J. Biol. Chem., 273, 3756-3764.

Neve, K.A., Seamans, J.K. \& Trantham-Davidson, H. (2004) Dopamine receptor signaling. J. Recept. Signal Tr. R., 24, 165-205.

Oleksy, A., Opalinski, L., Derewenda, U., Derewenda, Z.S. \& Otlewski, J. (2006) The molecular basis of RhoA specificity in the guanine nucleotide exchange factor PDZ-RhoGEF. J. Biol. Chem., 281, 3289132897.

Pollitt, S.K., Pallos, J., Shao, J., Desai, U.A., Ma, A.A., Thompson, L.M., Marsh, J.L. \& Diamond, M.I. (2003) A rapid cellular FRET assay of polyglutamine aggregation identifies a novel inhibitor. Neuron, 40, 685694.

Roze, E., Saudou, F. \& Caboche, J. (2008) Pathophysiology of Huntington's disease: from huntingtin functions to potential treatments. Curr. Opin. Neurol., 24, 497-503.

Roze, E., Bonnet, C., Betuing, S. \& Caboche, J. (2010) Huntington's disease. Adv. Exp. Med. Biol., 685, 45-63.

Seeman, P. (2010) Dopamine D2 receptors as treatment targets in schizophrenia. Clin. Schizophr. Relat. Psychoses, 4, 56-73.

Shirasaki, D.I., Greiner, E.R., Al-Ramahi, I., Gray, M., Boontheung, P., Geschwind, D.H., Botas, J., Coppola, G. et al. (2012) Network organization of the huntingtin proteomic interactome in mammalian brain. Neuron, 75, 41-57.

The Huntington's Disease Collaborative Research Group (1993) A novel gene containing a trinucleotide repeat that is expanded and unstable on Huntington's disease chromosomes. Cell, 72, 971-983.

Tourette, C., Li, B., Bell, R., O'Hare, S., Kaltenbach, L.S., Mooney, S.D. \& Hughes, R.E. (2014) A large scale Huntingtin protein interaction network implicates Rho GTPase signaling pathways in Huntington disease. J. Biol. Chem., 10, 6709-6726.

Vallone, D., Picetti, R. \& Borrelli, E. (2000) Structure and function of dopamine receptors. Neurosci. Biobehav. R., 24, 125-132.

Vonsattel, J.P., Myers, R.H., Stevens, T.J., Ferrante, R.J., Bird, E.D. \& Richardson, E.P. Jr (1985) Neuropathological classification of Huntington's disease. J. Neuropath. Exp. Neur., 44, 559-577.

Yin, H.H. \& Lovinger, D.M. (2006) Frequency-specific and D2 receptormediated inhibition of glutamate release by retrograde endocannabinoid signaling. Proc. Natl. Acad. Sci. USA, 103, 8251-8256. 